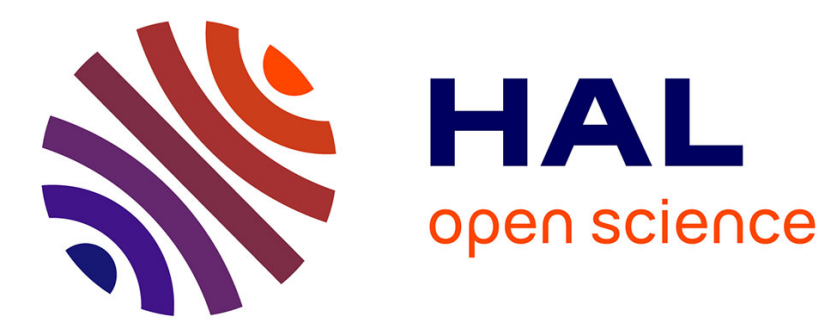

\title{
Robust Sub-10 nm Pattern of Standing Sugar Cylinders via Rapid "Microwave Cooking"
}

Yingjie Liao, Kangping Liu, Wen-Chang Chen, Bin Wei, Redouane Borsali

\section{To cite this version:}

Yingjie Liao, Kangping Liu, Wen-Chang Chen, Bin Wei, Redouane Borsali. Robust Sub-10 nm Pattern of Standing Sugar Cylinders via Rapid "Microwave Cooking". Macromolecules, 2019, 52 (22), pp.8751-8758. 10.1021/acs.macromol.9b01513 . hal-02954578

\section{HAL Id: hal-02954578 \\ https://cnrs.hal.science/hal-02954578}

Submitted on 22 Dec 2020

HAL is a multi-disciplinary open access archive for the deposit and dissemination of scientific research documents, whether they are published or not. The documents may come from teaching and research institutions in France or abroad, or from public or private research centers.
L'archive ouverte pluridisciplinaire HAL, est destinée au dépôt et à la diffusion de documents scientifiques de niveau recherche, publiés ou non, émanant des établissements d'enseignement et de recherche français ou étrangers, des laboratoires publics ou privés. 


\title{
Robust Sub-10 nm Pattern of Standing Sugar Cylinders via Rapid 'Microwave Cooking'
}

Yingjie Liao, ${ }^{a, b}$ Kangping Liu ${ }^{a}$, Wen-Chang Chen ${ }^{c}$, Bin Wei ${ }^{a}$ and Redouane Borsali $*^{b}$

a. Key Laboratory of Advanced Display and System Application, Ministry of Education, Shanghai University, Shanghai 200072, China

b. University Grenoble Alpes, CERMAV-CNRS, 38000 Grenoble, France.

c. Department of Chemical Engineering, National Taiwan University, Taipei 10617, Taiwan.

*Corresponding author: R. Borsali - e-mail: redouane.borsali@univ-grenoble-alpes.fr Keywords: carbohydrate, microwave heating, self-assembly, nanostructured thin films, grazingincidence small angle $\mathrm{X}$-ray scattering

\begin{abstract}
Block copolymer self-assembly is a widely used technique for obtaining many interesting nanostructures. Development of efficient and rapid processes for driving block copolymer selfassembly has remained a challenge. Microwave heating has attracted much attention in bioenergy production and food industry due to the advantages associated with dielectric heating effects. We report here a simple method (like microwave cooking) of microwave-heatinginduced microphase separation of a carbohydrate-based block copolymer, maltoheptaose-block-
\end{abstract}


polystyrene (MH-b-PS). Highly ordered and perpendicularly orientated $\mathrm{MH}$ cylinders $7 \mathrm{~nm}$ in diameter were formed in the PS matrix on a bare silicon wafer after annealed at $333 \mathrm{~K}$ for one second. The whole process is performed at a relatively low temperature under one atmosphere, and no solvent is needed during the process. The mechanism of this method is investigated by using grazing-incidence small angle X-ray scatting technique to make a detailed comparison with conventional thermal annealing process. The MH-b-PS cannot achieve self-assembly by conventional thermal annealing. In contrast, microwave energy can transfer directly to the polar MH blocks in the MH-b-PS thin films, which helps to make MH-b-PS self-assemble quickly. This study circumvents a major barrier to using carbohydrate-based block copolymer materials.

\section{Introduction}

Block copolymer (BCP) materials have attracted a lot of research interest since they can selfassemble into many useful nanostructures like hexagonal cylinders, cubic spheres, alternating lamellae, and bicontinuous gyroids. ${ }^{1,2}$ These nanostructures have exhibited a wide variety of applications in nanolithography, ${ }^{3,4}$ electronic and energy devices, ${ }^{5-9}$ nanoscale templating and patterning, ${ }^{10,11}$ which have led to important developments in the semiconductor and many other industries. To fully realize the enormous potential of BCP materials in these applications, however, many problems need to be addressed.

A main challenge is that the size of self-assembled $\mathrm{BCP}$ features is required to be extremely small in many applications. ${ }^{10,12-14}$ Developing BCPs with high Flory-Huggins interaction parameters $(\chi)$ and low degrees of polymerization $(\mathrm{N})$ is a strategy used by many people for obtaining a small feature size in BCP self-assembly. ${ }^{12}$ Carbohydrates have high incompatibility with synthetic polymers, so a BCP will have a high $\chi$ parameter if it is comprised of carbohydrates and synthetic polymers. Many carbohydrates-based BCPs have been synthesized 
successfully via click chemistry in our group 4, 11, 15, 16. By decreasing the degree of polymerization, the feature diameters of carbohydrate-based BCPs can go down to sub-10 nm. ${ }^{4}$, ${ }^{16}$ Maltoheptaose-block-polystyrene (MH-b-PS) is one of the carbohydrates-based BCPs which are capable of forming sub-10 nm scale cylindrical morphologies after solvent vapor annealing (SVA). ${ }^{16}$ A chemical structure of the MH-b-PS is shown in Figure 1. MH-b-PS can be synthesized through copper-catalyzed azide-alkyne cycloaddition of propargyl-functionalized maltoheptaose with azido end-functionalized polystyrene in dimethylformamide ${ }^{11,15,16}$. It has been demonstrated that MH-b-PS can be applied to non-volatile transistor devices, ${ }^{7}$ directed selfassembly and nanolithography. ${ }^{17}$ Although these carbohydrates-based BCP materials are promising for many applications ${ }^{7,16}$, it is difficult for them, e.g. MH-b-PS, to self-assemble with thermal annealing (TA) which is the method preferred by industry. And their SVA process takes a long time (about $24 \mathrm{~h}$ for MH-b-PS) ${ }^{16}$. Developing versatile annealing methods for achieving a fast self-assembly process with long-range ordering currently is an outstanding challenge for

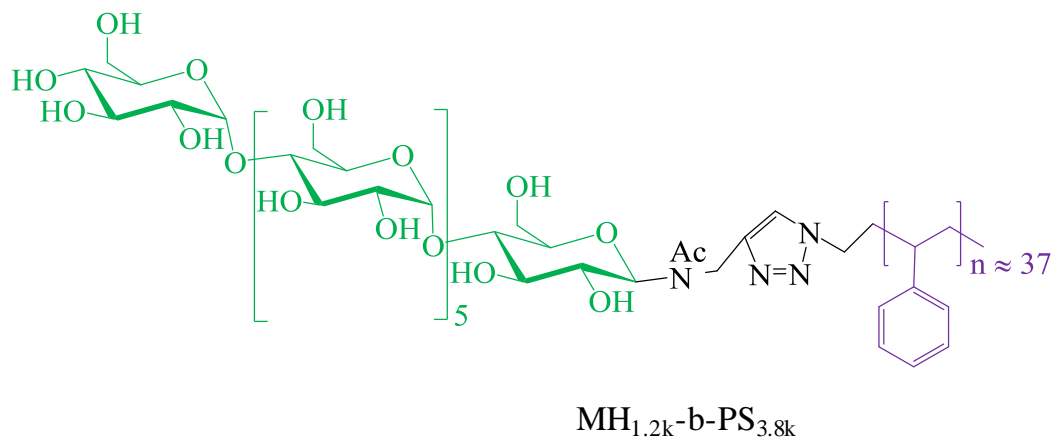

many BCP materials. ${ }^{18}$ As a consequence there is much research in this area.

Figure 1. Chemical structure of the MH-b-PS used in this study.

Many rapid ordering techniques, e.g. laser zone annealing, ${ }^{19}$ laser-spike, ${ }^{20}$ rapid thermal treatment, ${ }^{21}$ microwave annealing, ${ }^{22-28}$ etc., have been developed to replace conventional TA and SVA methods. Majewski et al. designed a laser heating method by putting a light-absorbing layer of germanium beneath poly(styrene)-b-poly(methyl methacrylate) (PS-b-PMMA) thin films. ${ }^{19}$ 
Highly localized laser heating produced photo thermal gradients which induced a thermophoretic force on morphological defects, and thus increased local ordering kinetics by at least 3 orders of magnitude compared to conventional oven annealing. Perego et al. used a so-called rapid thermal processing (RTP) technique to take advantage of the amount of solvent naturally trapped within the film during the spinning process and so obtained highly ordered lamellar grains of PS-bPMMA in a few seconds ${ }^{21}$. Buriak et al. used microwaves to heat the sample and annealing solvent simultaneously and obtained horizontally orientated cylinder-forming PS-b-PMMA and poly(styrene)-b-poly(2-vinylpyridine) (PS-b-P2VP) patterns in few minutes. ${ }^{22}$ Morris's group reported a microwave assisted annealing method for obtaining self-assembled PS-b-PMMA and polystyrene-b-polydimethylsiloxane (PS-b-PDMS) thin films in short times. ${ }^{23}$ They suggested that the polar molecules (polymethylmethacrylate and polydimethylsiloxane) could interact with the oscillating field resulting in dipole rotation which drives microphase separation of the PS-bPMMA and the PS-b-PDMS. However, Buriak et al. later determined that it is not the polar molecules but the microwave absorber which plays a dominate role in the fast self-assembly with the microwave annealing process. They also demonstrated that the annealing time can be reduced to 1 minute by placing a piece of high resistivity silicon wafer (a microwave absorber) in contact with the sample. ${ }^{24}$ Jonathan's group also reported that the block copolymer polystyrene-blockpoly(ethylene-co-butylene)-block-polystyrene is a poor microwave absorber, resulting in no change in the block copolymer morphology upon application of microwave energy. ${ }^{27}$ Our group has reported a fast ordering method termed high temperature solvent vapor annealing (HTSVA) ${ }^{25}$ The HTSVA provided an ideal binary solvent vapor environment at a high temperature using microwave energy that achieved self-assembly of the MH-b-PS in one second. In summary, researchers have tried to find an effective way to heat the medium (e.g. a rapid 
thermal processing system, a germanium layer, high resistivity silicon wafers or a high temperature solvent vapor environment) contacted with the target BCP materials. In these ways, energy could transfer easily to the target BCP materials through the medium and thus the $\mathrm{BCP}$ self-assembly process could finish in a short time.

The concept "microwave annealing" is found in many publications, but in these publications the microwave energy is used in different ways. There is no clear definition of "microwave annealing" until now. Here we would like to clarify the microwave approach used in this manuscript. It does not use a combination of solvent and heat like most publications, but use heat only. The microwave energy was utilized to heat the carbohydrate block maltoheptaose, residual moisture and the doped silicon substrates. While in our prior work ${ }^{25}$, a combination of solvent and heat was used for annealing. The microwave energy in our prior work was used to heat the annealing solvent only. Here we would like to probe how to transfer microwave energy directly and efficiently to carbohydrate-based BCP materials and make them self-assemble rapidly. We expect microwave heating can be used for heating carbohydrate-based BCP materials, because it is widely used in carbohydrate chemistry ${ }^{29}$, food preparation $^{30}$, bioenergy ${ }^{31}$, organic synthesis ${ }^{32}$ and so on. This unique heating mechanism provides many advantages, including a higher energy transfer efficiency, a shorter heating time to a set temperature, etc. that could benefit tremendously the self-assembly of carbohydrate-based BCP materials. Microwave heating is the reverse of conventional heating. Conventional heating (e.g. oven heating) transfers heat from an external heat source first to the surface of a material and then to the cooler interior regions by thermal conduction. Microwave heating, however, is a form of energy conversion rather than a form of heating, because electromagnetic energy is converted into heat directly in microwave heating ${ }^{33}$. In addition, the effect of microwave heating is almost instantaneous, which is different 
from conventional heating methods. This characteristic is of great interest for many practical applications since no time is spent waiting for the source to heat up or cool down ${ }^{32}$. Microwave heating displays a penetration depth (with penetration depth depending on dielectric constant and wavelength). Most likely this penetration depth is in the mm or higher range, so much thicker than the film thickness. What is more, microwave heating enables better control of the heating process itself and eliminates the risk of the sample surface overheating and possibly degrading (whilst the inner part has not yet been heated). This could be quite useful in improving the degree and range of ordering of whole BCP thin films.

2. Results and Discussion

In this study, atomic force microscopy (AFM) and grazing-incidence small-angle X-ray scattering (GISAXS) techniques have been used for investigating the microphase separation of

(a)

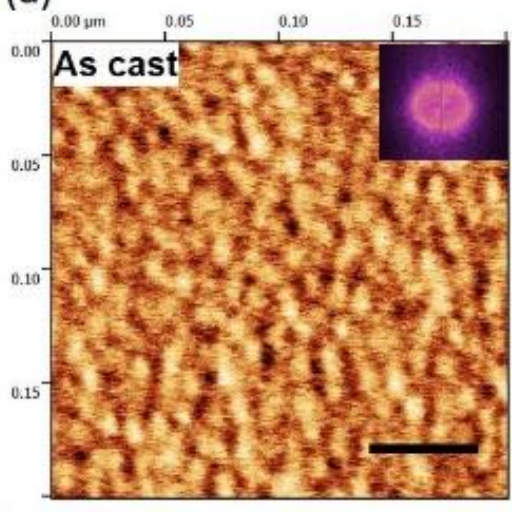

(d)

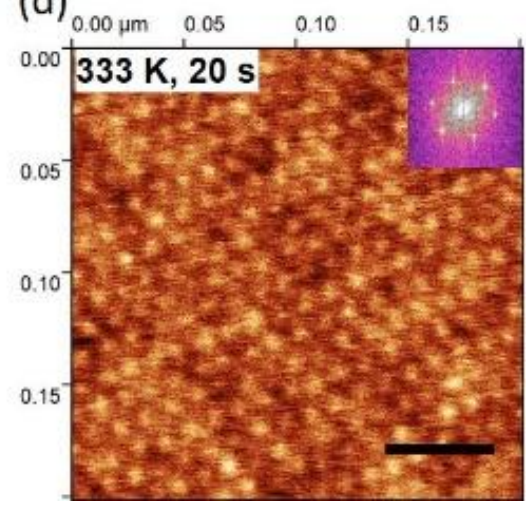

(b)

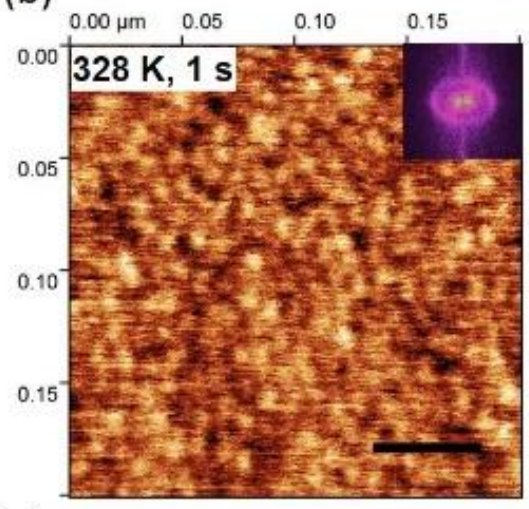

(e)

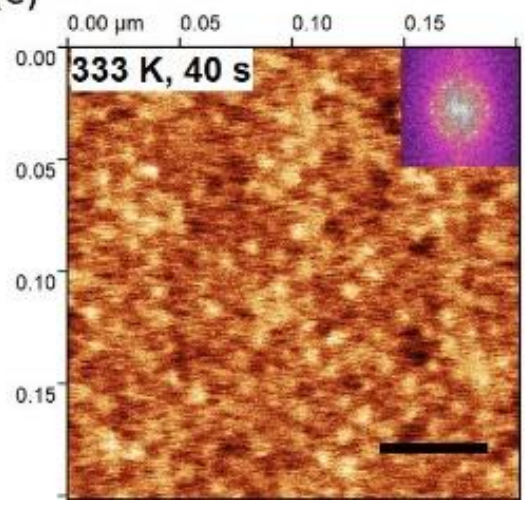

(c)

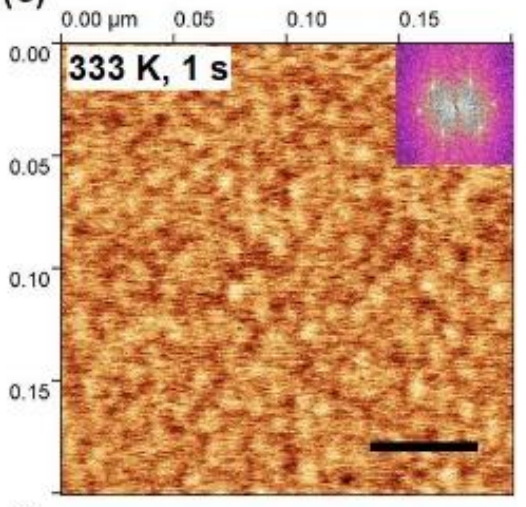

(f)

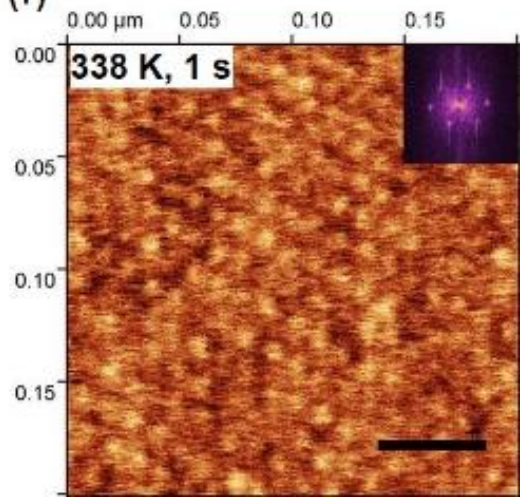


MH-b-PS thin films in the real and reciprocal space.

Figure 2. Atomic force microscopy phase images of the MH-b-PS thin films (a) before and (b-f) after microwave heating. The set temperature and corresponding duration time at the set

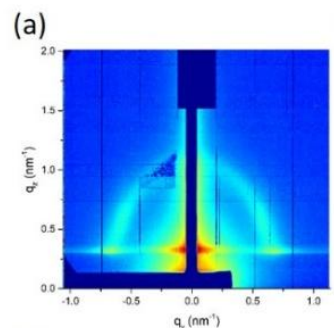

(d)

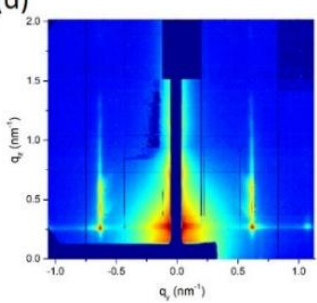

(b)

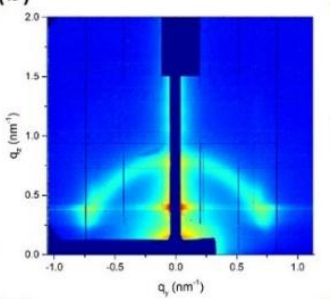

(e)

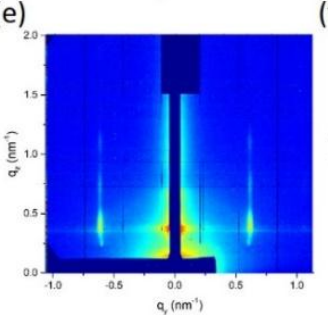

(c)

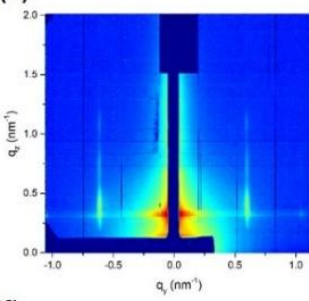

(f)

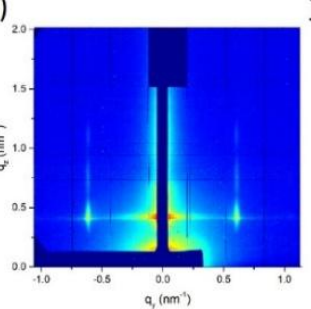

(g)

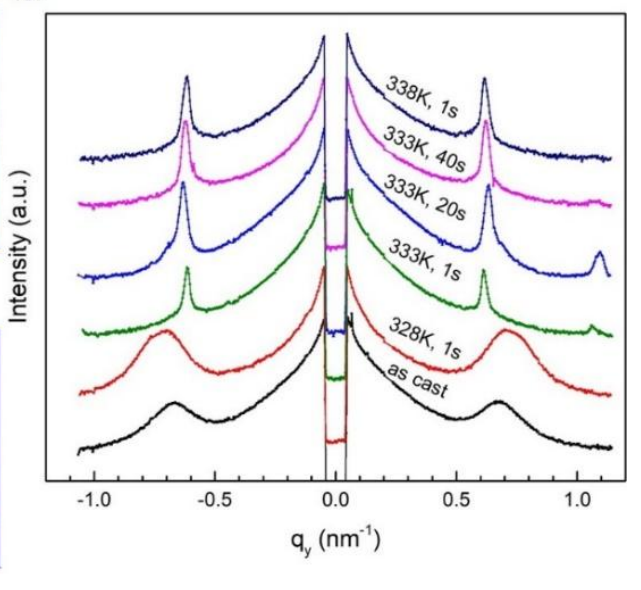

temperature are indicated on the images. The scale bars in the phase images represent $50 \mathrm{~nm}$.

The insets are 2D Fourier transfer images.

Figure 3. Grazing-incidence small-angle X-ray scattering (GISAXS) results of the MH-b-PS thin films on silicon wafers after microwave heating under different temperatures and times. (a-f) Corresponding GISAXS patterns of the MH-b-PS thin films shown in Figure 2. (g) Scans of the GISAXS patterns along the $\mathrm{q}_{\mathrm{y}}$ direction.

Figure 2 shows AFM phase images of MH-b-PS thin films under different microwave heating conditions, and Figure 3 presents corresponding GISAXS patterns and scans of the GISAXS patterns in the $\mathrm{q}_{\mathrm{y}}$ direction. The AFM phase image of the as cast MH-b-PS sample in Figure $2 \mathrm{a}$ exhibits disordered spherical morphology. Its GISAXS profile in Figure 3a shows two symmetrical Bragg rods at around -0.7 and $0.7 \mathrm{~nm}^{-1}$ linked by a so-called Debye-Scherrer ring, indicating that disordered spheres were formed in the as cast MH-b-PS thin film. This is 
consistent with what is observed in its AFM phase image. After microwave heating at $328 \mathrm{~K}$ for $1 \mathrm{~s}$, the AFM phase image (Figure 2b) of the MH-b-PS thin film does not show sensitive changes in terms of degree of ordering. Its GISAXS pattern in Figure $3 \mathrm{~b}$ is also similar to that of as cast sample in Figure 3a, except an increase in the intensity of the Bragg rods. This suggest that disordered spherical MH-b-PS nanostructures do not self-assemble into periodic nanostructure under this condition. When the set temperature is increased to $333 \mathrm{~K}$, the GISAXS pattern changes dramatically. As shown in Figure 3c, the Debye-Scherrer rings observed in Figure 3a and $3 \mathrm{~b}$ disappear and four symmetrical Bragg rods appear along the Yoneda band. The positions of the Bragg rods are at \pm 0.61 and $\pm 1.06 \mathrm{~nm}^{-1}$, which are consistent with characteristic pattern of a hexagonally packed array of cylinders normal to the surface. The AFM phase image in Figure $2 \mathrm{c}$ confirms the cylindrical morphology with a feature size about $8 \mathrm{~nm}$. The domain spacing is around $10.3 \mathrm{~nm}$, which is calculated from $\mathrm{q}^{*}=0.61 \mathrm{~nm}^{-1}$ by Bragg's equation. The two main Bragg rods at $\pm 0.61 \mathrm{~nm}^{-1}$ in Figure $3 \mathrm{c}$ can be assigned to the (10) reflection of the $2 \mathrm{D}$ hexagonal lattice formed by the MH cylinders. The other two rods at $\pm 1.06 \mathrm{~nm}^{-1}$ are just located at the scattering vectors of $\sqrt{3} q_{10}$ and are thus assigned to (11) reflections. Intensity profiles along the $\mathrm{q}_{\mathrm{y}}$ direction are shown in Figure 3g. A center-to-center distance of $11.8 \mathrm{~nm}$ can be calculated from the scattering vector of $q_{10}$. The intensity of both (10) and (11) rods are increased when extending the microwave heating time from $1 \mathrm{~s}$ to $20 \mathrm{~s}$ at $333 \mathrm{~K}$, see Figure $3 \mathrm{~d}$. The corresponding AFM phase image (Figure 2d) also exhibits an improvement in the degree of ordering. The trend towards a higher degree of ordering, however, changes when extending the microwave heating time at $333 \mathrm{~K}$. The AFM phase image of the MH-b-PS thin film in Figure 2e seems to some extent degrade after $40 \mathrm{~s}$ microwave irradiation. The intensity of diffractions in its GISAXS pattern in Figure 3e decreases as compared to Figure 3d. It is worth noting that the 
phase transition from spherical domains (as cast) to perpendicularly oriented cylindrical domains 
was fast. As the set temperature is increased to $338 \mathrm{~K}$, the GISAXS pattern in Figure $3 \mathrm{f}$ does not 
show sensitive changes as compared to Figure $3 \mathrm{c}$ at $333 \mathrm{~K}$, except that the (11) reflections 
become rather weak. This could be explained by the existence of a minimum in the form factor at 
the corresponding scattering vector. When the set temperature goes up to $343 \mathrm{~K}$, we found a 
smell of burnt sugar during the experiment. It is believed that the MH segment of the MH-b-PS 
caramelized under this condition. The working window for microwave irradiation induced self- 
assembly of the MH-b-PS is at around $333 \mathrm{~K}$ for a few seconds. This may due to a high efficient

(a)

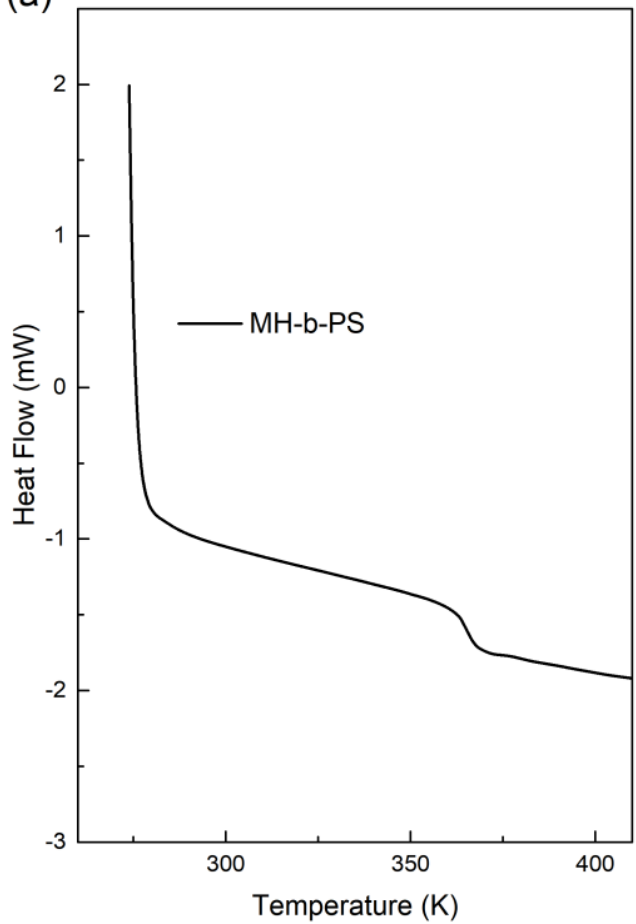

(b)

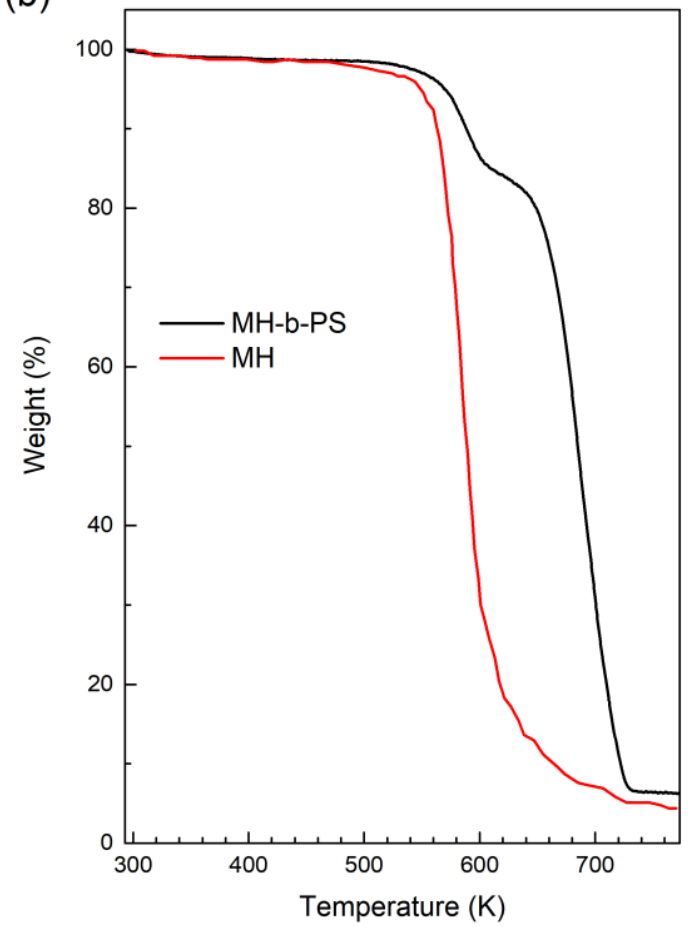

heating for the small volume of the MH-b-PS in the thin films, and probably could be solved by reducing the frequency of the microwaves. The full width at half maximum (FWHM) of the first order peak of as cast sample is $0.15 \mathrm{~nm}^{-1}$. After microwave heating at $328 \mathrm{~K}$ for $1 \mathrm{~s}$, the FWHM increases to about $0.27 \mathrm{~nm}^{-1}$, which implies the grain size of disordered nanostructures decreases under this condition. When the set temperature increases to $333 \mathrm{~K}$ and keep annealing for $1 \mathrm{~s}$, the FWHM decrease to $0.04 \mathrm{~nm}^{-1}$. It means cylindrical structures with a correlation length of $25 \mathrm{~nm}$ are formed in the MH-b-PS thin film. The FWHW at $333 \mathrm{~K}$ increases to about $0.06 \mathrm{~nm}^{-1}$ when the annealing time is extended to $20 \mathrm{~s}$ and even $40 \mathrm{~s}$, which means that the correlation length decreases at the beginning and then keep constant during microwave heating. When the set temperature goes up to $338 \mathrm{~K}$, the FWHM is still $0.06 \mathrm{~nm}^{-1}$. This suggests the correlation length dose not increase once the cylindrical structures are self-assembled in the thin films during microwave heating. 
Figure 4. (a) Differential scanning calorimetry (DSC) result of the MH-b-PS and (b) thermogravimetric analysis (TGA) of the MH-b-PS and the MH. 
Here the set temperature of microwave heating is around $333 \mathrm{~K}$, which is lower than the glass transition temperature of the MH-b-PS $\left(\mathrm{T}_{\mathrm{g}}=365 \mathrm{~K}\right.$, see Figure $\left.4 \mathrm{a}\right)$. It implies that the mechanism of microwave heating induced self-assembly of the MH-b-PS is different from that of

(a)

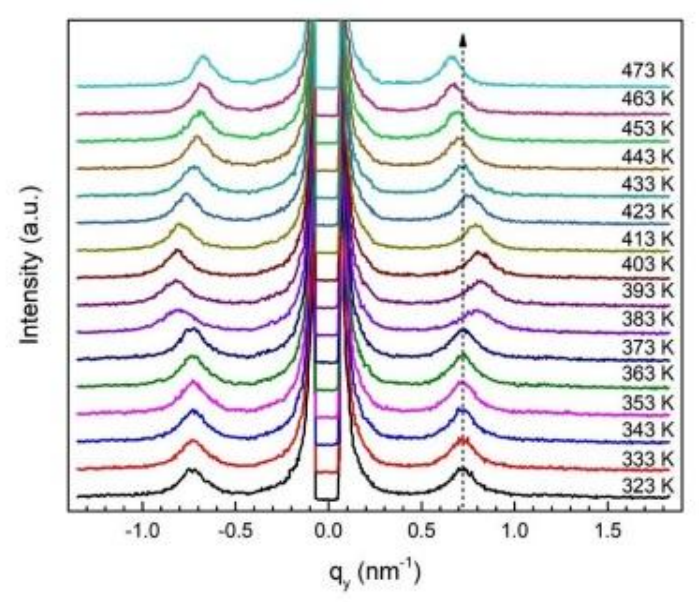

(b)
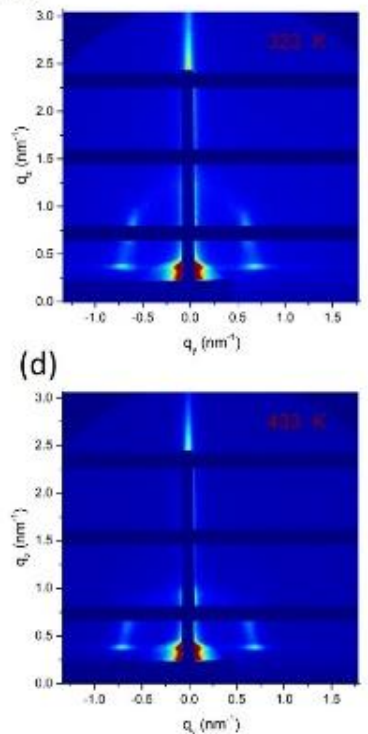

(c)
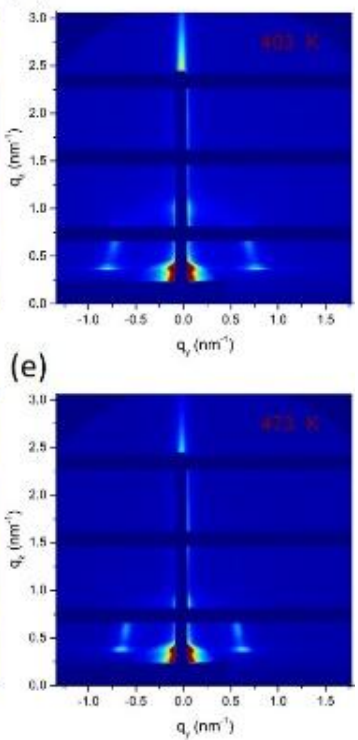

conventional heating induced self-assembly. Although the silicon wafer substrates used in this study have a relatively small size and low sheet resistance, they could also be heated by the microwaves and contribute to the self-assembly of the MH-b-PS through thermal annealing. Morris's group performed a study on the actual temperature of silicon substrates in a microwave heating system by attaching an independent thermometer to the silicon substrate ${ }^{26}$. They found the actual temperature of the silicon substrate did not exceed the set temperature and is even lower than the set temperature. This means the infrared sensor in the microwave heating system can provide a correct temperature measurement during microwave heating. In order to understand microwave heating induced self-assembly of MH-b-PS, we compare it with conventional heating by performing an in situ GISAXS measurement of MH-b-PS thin films on silicon wafers from $323 \mathrm{~K}$ to $473 \mathrm{~K}$. 
Figure 5. In situ GISAXS measurements of MH-b-PS thin films on silicon wafers using conventional heating at different temperatures. The annealing time is $3 \mathrm{~min}$ for all set temperatures. (a) Scans of the GISAXS patterns along the $\mathrm{q}_{\mathrm{y}}$ direction from $323 \mathrm{~K}$ to $473 \mathrm{~K}$. A vertical dash at $0.72 \mathrm{~nm}^{-1}$ is drawn for comparing the peak position. Representative GISAXS patterns are collected at (b) $323 \mathrm{~K}$, (c) $403 \mathrm{~K}$, (d) $433 \mathrm{~K}$ and (e) $473 \mathrm{~K}$.

Figure 5 shows $\mathrm{q}_{\mathrm{y}}$ scans of the GISAXS patterns from $323 \mathrm{~K}$ to $473 \mathrm{~K}$ and 4 representative GISAXS patterns at 323, 403, 433 and $473 \mathrm{~K}$, respectively. As presented in Figure 5a, all the GISAXS patterns exhibit only one pair of Bragg rods at around $\mathrm{q}_{\mathrm{y}}=-0.72$ and $0.72 \mathrm{~nm}^{-1}$ during the whole heating process. Neither the position nor the shape of the Bragg rods change from 323 K to $373 \mathrm{~K}$, which suggests MH-b-PS chains do not get enough mobility necessary to form any nanostructure in this temperature range. This can also be explained by the $\mathrm{T}_{\mathrm{g}}$ of MH-b-PS which is around $365 \mathrm{~K}$ (see Figure 4a). After the heating temperature rises to $373 \mathrm{~K}$, we find some effect of temperature on the GISAXS patterns. The position of the Bragg rods shifts towards $\mathrm{q}_{\mathrm{y}}=-$ 0.80 and $0.80 \mathrm{~nm}^{-1}$ from $383 \mathrm{~K}$ to $403 \mathrm{~K}$, which indicates the domain space of MH-b-PS nanostructure decreases and the MH-b-PS thin film has a trend towards self-assembly. However, no higher order Bragg rods occur and the Bragg rods become broader. That means the MH-b-PS thin films do not achieve self-assembly between $383 \mathrm{~K}$ and $403 \mathrm{~K}$. As the temperature continues to increase, the position of the Bragg rods firstly goes back to $q_{y}=-0.72$ and $0.72 \mathrm{~nm}^{-1}$ at $433 \mathrm{~K}$ and then shifts to $\mathrm{q}_{\mathrm{y}}=-0.66$ and $0.66 \mathrm{~nm}^{-1}$ at $473 \mathrm{~K}$. The GISAXS patterns at 403,433 and $473 \mathrm{~K}$ (see Figure 5b, c, d, e) are quite similar to the one at $323 \mathrm{~K}$ in terms of density distribution. All of this evidence suggests that the MH-b-PS thin film does not self-assemble in this heating process even when the temperature is increased to $473 \mathrm{~K}$. We thus conclude that it is quite difficult to make MH-b-PS self-assemble by conventional heating. In comparison, MH-b-PS thin 
films can self-assemble at $333 \mathrm{~K}$ into well-ordered cylindrical structures under microwave irradiation as shown before. This indicates that the main mechanism of microwave heating induced ultra-fast self-assembly of MH-b-PS is possibly not due to the heating of the silicon substrate.

Figure 6. GISAXS patterns of MH-b-PS thin films on fused silica substrates (a) before and after microwave heating at (b) $328 \mathrm{~K}$ for $1 \mathrm{~s}$, (c) $333 \mathrm{~K}$ for $1 \mathrm{~s}$, (d) $333 \mathrm{~K}$ for $20 \mathrm{~s}$, (e) $333 \mathrm{~K}$ for $40 \mathrm{~s}$, (f) $338 \mathrm{~K}$ for $1 \mathrm{~s}$. (g) Scans of the GISAXS patterns along the $\mathrm{q}_{\mathrm{y}}$ direction.

To eliminate the effect of silicon substrates in the microwave heating, we replaced them with
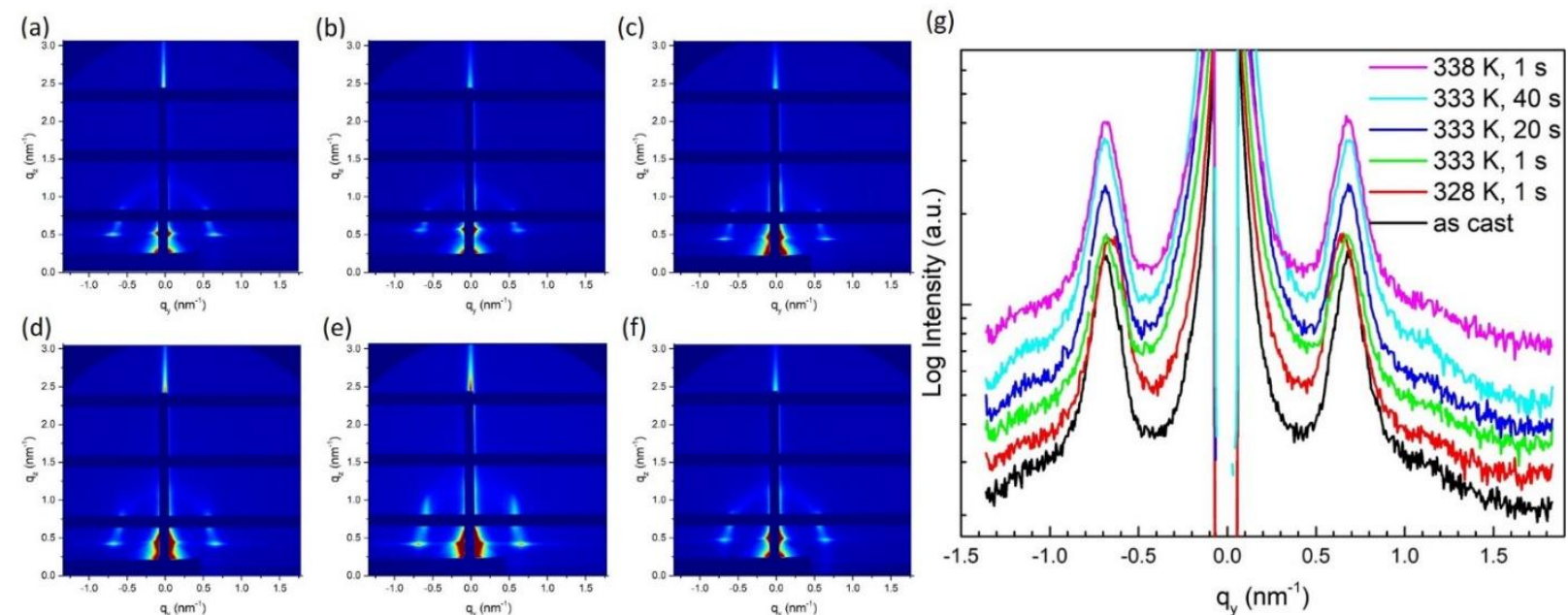

microwave transparent fused silica substrates. These fused silica substrates cannot be heated by microwaves, so microwaves will work only on the MH-b-PS thin films. These MH-b-PS samples were annealed with the same microwave heating parameters for comparison and then characterized by GISAXS. As presented in Figure 6a, the as cast MH-b-PS on fused silica substrate has a similar GISAXS pattern to the as cast MH-b-PS on silicon wafer. The positions of Bragg rods in both GISAXS patterns are around -0.7 and $0.7 \mathrm{~nm}^{-1}$. So the effect of different surfaces of the two kind of substrates on the self-assembly of MH-b-PS before annealing could be ignored in this comparison. After microwave heating at $328 \mathrm{~K}$ for $1 \mathrm{~s}$, the GISAXS pattern of 
the MH-b-PS thin film on fused silica substrate does not show sensitive differences in the density distribution. The GISAXS pattern does not change at $333 \mathrm{~K}$ for $1 \mathrm{~s}$. When the microwave heating time was extended to $20 \mathrm{~s}$ at $333 \mathrm{~K}$, we found weak secondary order scatting peaks at around 1.1 and $1.1 \mathrm{~nm}^{-1}$ from the $\mathrm{q}_{\mathrm{y}}$ scan of the GISAXS pattern in Figure 6d. The two secondary order scattering peaks enhance in Figure 6e when the microwave heating time is increased to $40 \mathrm{~s}$ at $333 \mathrm{~K}$, but they are not as strong as the case of the MH-b-PS thin film on the silicon wafer. These results indicate that microwave energy can be absorbed by the MH-b-PS thin film and used for driving the self-assembly of MH-b-PS. However, the degree of ordering of the selfassembled MH-b-PS thin film on fused silica substrates is lower than the MH-b-PS thin film on silicon substrate. One possible explanation is that microwave-heated $\mathrm{MH}$ block is minor which has not enough ability to drive completely the whole MH-b-PS molecule to self-assembly. While the chain mobility of the PS matrix can be increased with the help of microwave-heated silicon substrate. MH-b-PS can self-assemble easier on silicon substrates than on fused silica substrates during microwave heating. It has been demonstrated above that the MH-b-PS cannot selfassemble with only conventional heating. So we think that the heating effect on both the MH-bPS thin film and the silicon substrate is critical for achieving ultra-fast self-assembly of the MHb-PS by microwave heating.

It is important to get insight on how the MH-b-PS thin film absorbs microwave energy. There are at least three components in the MH-b-PS thin films: MH-b-PS, residual solvent and moisture. The two segments of the MH-b-PS, polystyrene and maltoheptaose, have different dielectric properties. Polystyrene is a nonpolar molecule since it has a symmetrical structure. So it is transparent to microwaves. But the maltoheptaose in the present study is obtained from a saturated sugar $\beta$-cyclodextrin which has a high dielectric constant ${ }^{34}$. As shown in Figure 1 , there 
are 7 units of glucose with 22 polar oxygen-hydrogen bonds in the maltoheptaose. These hydroxyl groups are in an asymmetric geometry, so maltoheptaose is overall highly polar. The hydroxyl groups in saccharides like maltoheptaose could create a high shear environment by attempting to continuously reorient in the electromagnetic radiation's oscillating field, which is similar to the behavior of the water dipole. Dependent on the frequency, the dipole may move in time with the field, lag behind it or remain apparently unaffected ${ }^{35}$. When the dipole lags behind the field then interactions between the dipole and the field lead to an energy loss by heating, the extent of which is dependent on the phase difference of these fields. The dipole-dipole interactions could also exist in the maltoheptaose domains during microwave heating and contribute to the self-assembly of MH-b-PS. In addition, hydrogen bonds and hydroxyl-water interactions also play a significant role in high sugar, maltodextrin, starch hydrolysate, and lactose-like disaccharide-based food formulations ${ }^{36}$. Since microwaves act only on the $\mathrm{MH}$ segment, the heat produced in the MH domain transfers to the PS segments as microwave heating time is extended. The MH segment is a minority component of the MH-b-PS, so it is difficult for MH-b-PS to have enough chain mobility to achieve well-ordered self-assembly without the heating of silicon substrates.

Residual solvent and moisture inside the MH-b-PS thin films could be heated together with the MH-b-PS by microwaves during the annealing process. Although both of them could be removed after reaching a specific temperature, they may contribute to an increase in the temperature of MH-b-PS thin films and influence the self-assembly of MH-b-PS before their removal. The solvent used for fabricating MH-b-PS thin films is anisole. Because anisole is a nonpolar solvent and does not absorb energy from microwaves, the effect of the residual anisole inside MH-b-PS thin films is negligible. On the contrary, depending on the hydrophilic property and relative 
humidity of the surrounding environment, saccharide polymers, like maltoheptaose, usually contain several percent moisture which can be heated by microwaves. In order to estimate the amount of moisture inside MH-b-PS thin films and investigate physical and chemical processes of the MH-b-PS as the temperature increases, we performed thermogravimetric analysis (TGA). Usually, the moisture content of a material is eliminated when the temperature goes up to $373 \mathrm{~K}$. As shown in the TGA curves (see Figure $4 b$ ), the weight of the MH-b-PS decreases just 1\% from room temperature to $373 \mathrm{~K}$. This TGA curve stays flat up to $473 \mathrm{~K}$, which proves that there is only a very small amount of moisture (about $1.5 \mathrm{wt} \%$ ) inside the MH-b-PS. Since the PS segment of the MH-b-PS is hydrophobic, the moisture could only adhere to the hydrophilic segment of the MH-b-PS (the MH segment). This assumption is proved from the TGA curve of the MH. As compared to the TGA curve of MH-b-PS, the TGA curve of MH shows the same characteristic from room temperature to $473 \mathrm{~K}$. This means that the moisture lost from the $\mathrm{MH}-$ b-PS is originally involved in the $\mathrm{MH}$ segment as the temperature is elevated from room temperature to $473 \mathrm{~K}$. It should be noted that this small amount of moisture inside the $\mathrm{MH}$ segment is not fully eliminated at the annealing temperatures ( $328 \mathrm{~K}, 333 \mathrm{~K}$ and $338 \mathrm{~K})$. Some moisture (less than 1\%) is still trapped inside the MH segment until 373K. We think the moisture as a dielectric material could contribute to an increase in the temperature of the MH-b-PS during the annealing process, but it does not play a main role in driving the self-assembly of the MH-bPS due to its very small amount.

\section{Conclusion}

It is demonstrated here that microwave energy can be absorbed directly by thin films of the carbohydrate-based BCP material MH-b-PS and then drives the MH-b-PS self-assembly in a few seconds without need of any solvents. Perpendicularly orientated cylinder-forming MH-b-PS 
nanopatterns with $7 \mathrm{~nm}$ feature size and long-range ordering across the whole thin film were obtained in one second at $333 \mathrm{~K}$ using this microwave heating method. The rotation of the $\mathrm{MH}$ dipole under microwave irradiation is the main reason for achieving ultra-fast self-assembly of the MH-b-PS. The small amount of moisture inside the MH-b-PS is also helpful for the effective microwave heating of MH-b-PS. In addition, the silicon substrate is indispensable in the microwave heating induced self-assembly of the MH-b-PS, because it can help enhance the flexibility of the PS chain of the MH-b-PS. In total, the microwave heating method is much more efficient than both conventional heating and solvent vapor annealing in driving the microphase separation of MH-b-PS. It is expected that this microwave heating method can be applied to other carbohydrate-based materials, morphologies, and directed self-assembly strategies and would be useful in microelectronic industries.

\section{Experimental Section}

Thin film preparation: The Si wafers (p-type, B-doped, orientation $=100 \pm 0.5^{\circ}, \rho=1-50 \Omega \mathrm{cm}$ ) were purchased from Siltronix and then diced into approximately $1.0 \mathrm{~cm} \times 0.5 \mathrm{~cm}$ pieces. In order to avoid the effect of difference in the resistance of silicon substrates, all the silicon substrates used are from the same piece of silicon wafer. The silicon pieces were degreased in an ultrasonic bath of acetone for $20 \mathrm{~min}$, rinsed in ethanol, and then dried by a nitrogen stream. A radio-frequency oxygen plasma (ION3 KHZ plasma system, PVA TePla) treatment at 0.77 torr, $41.3 \mathrm{cc} / \mathrm{min}, 40 \mathrm{~W}$ of power for $10 \mathrm{~min}$ was performed to render hydrophilic surfaces for the silicon substrates. Preparation of MH-b-PS thin films was performed immediately after the plasma treatment for the silicon substrates. The fused silica substrates were ordered from Edmund optics. The MH-b-PS thin films on fused silica substrates were fabricated using the same parameters as the MH-b-PS thin films on Si wafers. 
Anisole (purity > 99\%) was acquired from Sigma-Aldrich and used without further purification. The diblock copolymer MH-b-PS was weighed and dissolved in anisole at room temperature by stirring overnight. The concentration of the $\mathrm{MH}-\mathrm{b}-\mathrm{PS}$ solutions is $20 \mathrm{mg} / \mathrm{ml}$ unless otherwise specified. A volume of $40 \mu \mathrm{l}$ of the MH-b-PS solution was dropped onto a plasma-treated silicon substrate and spin coated (spin coater model: SPS Spin 150) at 2000 RPM for 1 min under ambient conditions at room temperature. The film thickness is around $40 \mathrm{~nm}$. The spin-coated MH-b-PS films were transferred for microwave heating after drying in air.

'Microwave cooking': A commercial microwave synthesizer (Initiator 2.5, Biotage) was used as a microwave heating source for the annealing process. The Biotage reactor measures the temperature of the samples by an infrared sensor (accuracy: $5 \mathrm{~K}$ ) at the bottom of the microwave cavity. Since the vials used in our experiments are transparent for microwaves, the temperature detected by the infrared sensor is coming from the sample. The measurement of the temperature is an in-situ measurement. The MH-b-PS sample was put into a reaction vial and then heated with $2.45 \mathrm{GHz}$ microwave energy to reach the target temperature. The target temperature was kept for a given annealing time with the help of the temperature feedback-control system of the microwave synthesizer. The vial was then cooled down to $318 \mathrm{~K}$ with air flow. The whole heating and cooling process took approximately one minute, which depends on the target temperature. The output microwave power, the temperature of the vial and the pressure inside of the vial were recorded by the microwave synthesizer.

Atomic force microscopy measurements: Phase images were collected by an atomic force microscope (Agilent 5500, Agilent Technologies) in tapping mode. Silicon cantilevers were purchased from Budget Sensors with a resonant frequency of $150 \mathrm{kHz}$ and a force constant of 7.4 $\mathrm{N} / \mathrm{m}$. 
Grazing-incidence small-angle X-ray scattering (GISAXS): GISAXS measurements were performed on the CRG-BM02 beamline at the European Synchrotron Radiation Facility in Grenoble. GISAXS experiments used photo energies of 11 and $16 \mathrm{keV}$. The samples were positioned on a 6 circle-goniometer, allowing an accurate alignment of the patterned area with respect to the incident beam direction. The GISAXS patterns were recorded after $10 \mathrm{~s}$ irradiation on a XPAD 2D pixel detector placed at a distance of $1880 \mathrm{~mm}$ from the sample. The intense incident and reflected beams were stopped by a $2 \mathrm{~mm}$ wide Ta strip. A photomultiplier with a removable kapton foil was placed in front of the beam stop chamber to adjust sample position and measure rough reflectivity curves to check the Si critical angle. The incident angle was chosen between the critical angles of the polymer $\left(0.13^{\circ}\right)$ and the Si substrate $\left(0.18^{\circ}\right)$. With the incident beam propagating along the $\mathrm{x}$ direction, the GISAXS images were recorded in the $\left(\mathrm{q}_{\mathrm{y}}\right.$, $\mathrm{q}_{\mathrm{z}}$ ) plane where $\mathrm{q}_{\mathrm{y}}$ and $\mathrm{q}_{\mathrm{z}}$ are the components of the scattering vector, related to the in-plane angle $2 \theta_{\mathrm{f}}$ and out-plane angle $\alpha_{\mathrm{f}}$. The in situ GISAXS measurement was performed with a DHS 900 hot stage and TCU 150 temperature control unit. All the GISAXS patterns in the study were normalized to the incident beam intensity monitored by a front photomultiplier.

Thermogravimetric analysis (TGA) and Differential scanning calorimetry (DSC): TGA was conducted on a thermogravimetric analyzer (Setaram Instruments) using a ramp rate of $283 \mathrm{~K}$ per minute up to $773 \mathrm{~K}$ under nitrogen gas. DSC experiments were performed on a Q200 (TA instruments) under a nitrogen purge and at a heating rate of $293 \mathrm{~K}$ per minute. Data are reported on the second heat.

\section{Corresponding Author}

*Prof. Redouane BORSALI, 
CERMAV, BP 53, 38041 Grenoble cedex 9, France

E-mail: borsali@cermav.cnrs.fr

Tel: +33 (0)4 76037627 - fax : +33(0)4 76037629

\section{ACKNOWLEDGMENT}

This work was partially supported by the CNRS, the European project "GreeNanoFilms" which has received funding from the European Union Seventh Framework Program (FP7/20072013) under grant agreement No. 603519, and partially supported by the ANR project "Sweetmemory" (ANR-14-CE08-0021) and Carnot PolyNat Institut. This work is also supported by the program of Youth Eastern Scholar from Shanghai Municipal Education Commission. The authors are grateful to Prof. Robert Pecora (Stanford University, CA, USA) for helpful discussions. The authors thank ESRF for beam time allocation and thank D2AM staff Nathalie Boudet for her help in GISAXS measurement.

\section{Supporting Information}

Figures S1-S7.

\section{REFERENCES}

1. Albert, J. N. L.; Epps III, T. H. Self-assembly of block copolymer thin films. Mater Today 2010, 13 (6), 24-33.

2. Matsen, M. W. Effect of Architecture on the Phase Behavior of AB-Type Block Copolymer Melts. Macromolecules 2012, 45 (4), 2161-2165.

3. Mansky, P.; haikin, P.; Thomas, E. L. Monolayer films of diblock copolymer microdomains for nanolithographic applications. J. Mater. Sci. 1995, 30 (8), 1987-1992.

4. Cushen, J. D.; Otsuka, I.; Bates, C. M.; Halila, S.; Fort, S.; Rochas, C.; Easley, J. A.; Rausch, E. L.; Thio, A.; Borsali, R.; Willson, C. G.; Ellison, C. J. Oligosaccharide/SiliconContaining Block Copolymers with $5 \mathrm{~nm}$ Features for Lithographic Applications. ACS Nano 2012, 6 (4), 3424-3433. 
5. Hung, C.-C.; Chiu, Y.-C.; Wu, H.-C.; Lu, C.; Bouilhac, C.; Otsuka, I.; Halila, S.; Borsali, R.; Tung, S.-H.; Chen, W.-C. Conception of stretchable resistive memory devices based on nanostructure-controlled carbohydrate-block-polyisoprene block copolymers. Adv. Funct. Mater. 2017, 27 (13), 1606161.

6. Yoo, H. G.; Byun, M.; Jeong, C. K.; Lee, K. J. Performance enhancement of electronic and energy devices via block copolymer self-assembly. Adv. Mater. 2015, 27 (27), 3982-98.

7. Chiu, Y.-C.; Otsuka, I.; Halila, S.; Borsali, R.; Chen, W.-C. High-performance nonvolatile transistor memories of pentacence using the green electrets of sugar-based block copolymers and their supramolecules. Adv. Funct. Mater. 2014, 24 (27), 4240-4249.

8. Darling, S. B. Block copolymers for photovoltaics. Energy \& Environmental Science 2009, $2(12), 1266$.

9. Wang, M.; Wudl, F. Top-down meets bottom-up: organized donor-acceptor heterojunctions for organic solar cells. J. Mater. Chem. 2012, 22 (46), 24297-24314.

10. Fasolka, M. J.; Mayes, A. M. Block copolymer thin films: Physics and applications. Annu. Rev. Mater. Res. 2001, 31, 323-355.

11. Aissou, K.; Halila, S.; Fort, S.; Borsali, R.; Baron, T. Thin films organized in nanodomains on the basis of copolymers having polysaccharide blocks for applications in nanotechnology. WO2012013911 A1, 2016.

12. Sinturel, C.; Bates, F. S.; Hillmyer, M. A. High chi-Low N Block Polymers: How Far Can We Go? ACS Macro Letters 2015, 4 (9), 1044-1050.

13. Kim, J. K.; Yang, S. Y.; Lee, Y.; Kim, Y. Functional nanomaterials based on block copolymer self-assembly. Prog. Polym. Sci. 2010, 35 (11), 1325-1349.

14. Tseng, Y.-C.; Darling, S. B. Block Copolymer Nanostructures for Technology. Polymers 2010, 2 (4), 470-489.

15. Aissou, K.; Otsuka, I.; Rochas, C.; Fort, S.; Halila, S.; Borsali, R. Nano-Organization of Amylose-b-Polystyrene Block Copolymer Films Doped with Bipyridine. Langmuir 2011, 27 (7), 4098-4103.

16. Otsuka, I.; Tallegas, S.; Sakai, Y.; Rochas, C.; Halila, S.; Fort, S.; Bsiesy, A.; Baron, T.; Borsali, R. Control of $10 \mathrm{~nm}$ scale cylinder orientation in self-organized sugar-based block copolymer thin films. Nanoscale 2013, 5 (7), 2637-41.

17. Otsuka, I.; Nilsson, N.; Suyatin, D. B.; Maximov, I.; Borsali, R. Carbohydrate-based block copolymer systems: directed self-assembly for nanolithography applications. Soft Matter 2017, 13 (40), 7406-7411.

18. Majewski, P. W.; Yager, K. G. Rapid ordering of block copolymer thin films. J. Phys.Condes. Matter 2016, 28 (40), 37.

19. Majewski, P. W.; Yager, K. G. Millisecond Ordering of Block Copolymer Films via Photothermal Gradients. ACS Nano 2015, 9 (4), 3896-3906.

20. Jiang, J.; Jacobs, A. G.; Wenning, B.; Liedel, C.; Thompson, M. O.; Ober, C. K. Ultrafast Self-Assembly of Sub-10 nm Block Copolymer Nanostructures by Solvent-Free HighTemperature Laser Annealing. ACS Appl. Mater. Interfaces 2017, 9 (37), 31317-31324.

21. Perego, M.; Ferrarese Lupi, F.; Ceresoli, M.; Giammaria, T. J.; Seguini, G.; Enrico, E.; Boarino, L.; Antonioli, D.; Gianotti, V.; Sparnacci, K.; Laus, M. Ordering dynamics in symmetric PS-b-PMMA diblock copolymer thin films during rapid thermal processing. Journal of Materials Chemistry C 2014, 2 (32), 6655-6664. 
22. Zhang, X.; Harris, K. D.; Wu, N. L. Y.; Murphy, J. N.; Buriak, J. M. Fast assembly of ordered block copolymer nanostructures through microwave annealing. ACS Nano 2010, 4 (11), 7021-7029.

23. Borah, D.; Senthamaraikannan, R.; Rasappa, S.; Kosmala, B.; Holmes, J. D.; Morris, M. A. Swift nanopattern formation of PS-b-PMMA and PS-b-PDMS block copolymer films using a microwave assisted technique. ACS Nano 2013, 7 (8), 6583-6596.

24. Jin, C.; Murphy, J. N.; Harris, K. D.; Buriak, J. M. Deconvoluting the mechanism of microwave annealing of block copolymer thin films. ACS Nano 2014, 8 (4), 3979-3991.

25. Liao, Y.; Chen, W.-C.; Borsali, R. Carbohydrate-Based block copolymer thin films: ultrafast nano-organization with $7 \mathrm{~nm}$ resolution using microwave energy. Adv. Mater. 2017, 29 (35), 1701645-n/a.

26. Mokarian-Tabari, P.; Cummins, C.; Rasappa, S.; Simao, C.; Torres, C. M. S.; Holmes, J. D.; Morris, M. A. Study of the Kinetics and Mechanism of Rapid Self-Assembly in Block Copolymer Thin Films during Solvo-Microwave Annealing. Langmuir 2014, 30 (35), 10728-10739.

27. Toolan, D. T. W.; Adlington, K.; Isakova, A.; Kalamiotis, A.; Mokarian-Tabari, P.; Dimitrakis, G.; Dodds, C.; Arnold, T.; Terrill, N. J.; Bras, W.; Hermida Merino, D.; Topham, P. D.; Irvine, D. J.; Howse, J. R. Selective molecular annealing: in situ small angle X-ray scattering study of microwave-assisted annealing of block copolymers. Phys. Chem. Chem. Phys. 2017, 19 (31), 20412-20419.

28. Qiang, Z.; Ye, C.; Lin, K.; Becker, M. L.; Cavicchi, K. A.; Vogt, B. D. Evolution in surface morphology during rapid microwave annealing of PS-b-PMMA thin films. Journal of Polymer Science Part B: Polymer Physics 2016, 54 (15), 1499-1506.

29. Das, S. K. Application of Microwave Irradiation in the Synthesis of Carbohydrates. Synlett 2004, 2004 (06), 915-932.

30. Icier, F.; Baysal, T. Dielectrical properties of food materials - 1: Factors affecting and industrial uses. Critical Reviews in Food Science and Nutrition 2004, 44 (6), 465-471.

31. Kostas, E. T.; Beneroso, D.; Robinson, J. P. The application of microwave heating in bioenergy: A review on the microwave pre-treatment and upgrading technologies for biomass. Renewable and Sustainable Energy Reviews 2017, 77, 12-27.

32. Kappe, C. O. Controlled Microwave Heating in Modern Organic Synthesis. Angewandte Chemie International Edition 2004, 43 (46), 6250-6284.

33. Motasemi, F.; Afzal, M. T. A review on the microwave-assisted pyrolysis technique. Renewable and Sustainable Energy Reviews 2013, 28, 317-330.

34. Sakurai, M.; Kitagawa, M.; Hoshi, H.; Inoue, Y.; Chûjô, R. A molecular orbital study of cyclodextrin (cyclomalto-oligosaccharide) inclusion complexes. III, dipole moments of cyclodextrins in various types of inclusion complex. Carbohydr. Res. 1990, 198 (2), 181-191.

35. English, N. J.; MacElroy, J. M. D. Molecular dynamics simulations of microwave heating of water. The Journal of Chemical Physics 2003, 118 (4), 1589-1592.

36. Roebuck, B. D.; Goldblith, S. A.; Westphal, W. B. Dielectric Properties of CarbohydrateWater Mixtures at Microwave Frequencies. J. Food Sci. 1972, 37 (2), 199-204. 


\section{Table of Contents}

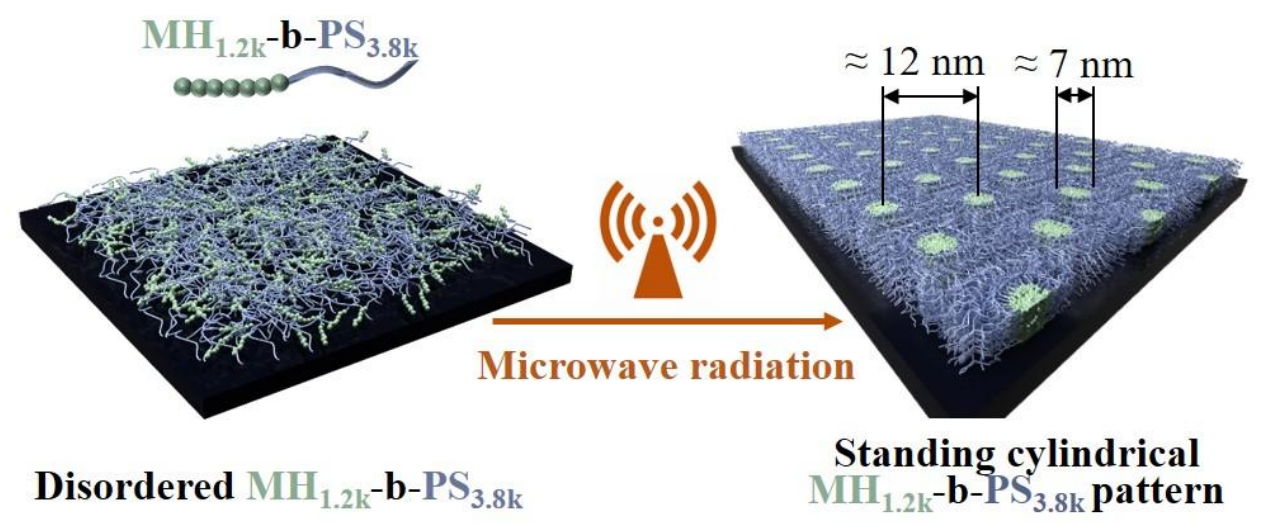

